\title{
Solving a class of Robin problems in simply connected regions via integral equations with a generalized Neumann kernel
}

\author{
Shwan H.H. Al-Shatri ${ }^{a}$, Ali H.M. Murid ${ }^{\mathrm{b}, \mathrm{c},}$, Munira Ismail ${ }^{\mathrm{b}}$ \\ a Department of Physics, College of Education, Charmo University, 46023 Chamchamal, Sulaimani, \\ Kurdistan, Iraq \\ b Department of Mathematical Sciences, Faculty of Science, Universiti Teknologi Malaysia, Johor, Malaysia \\ c UTM Centre for industrial and Applied Mathematics, \\ Ibnu Sina Institute for Scientific and Industrial Research, Universiti Teknologi Malaysia, Johor, Malaysia
}

*Corresponding author, e-mail: alihassan@utm.my

Received 22 Aug 2014

Accepted 21 May 2017

\begin{abstract}
This paper presents a new boundary integral equation method for the solution of a class of Robin problems in bounded and unbounded simply connected regions. We show how to reformulate the Robin problems as RiemannHilbert problems which lead to systems of integral equations, related differential equations, and normalizing conditions that give rise to unique solutions. Numerical results on several test regions are presented to illustrate the solution technique for the Robin problems when the boundaries are sufficiently smooth.
\end{abstract}

KEYWORDS: Riemann-Hilbert problem

\section{INTRODUCTION}

There are many applications of Laplacian differential operator related to physical geodesy, electromagnetic, measurement ${ }^{1,2}$, and to specific boundary problems such as Dirichlet problem and Neumann problem ${ }^{3}$. The applications of the mixed boundary value problem in potential theory can be found in Ref. 4. A mixed boundary value problem has mixed Dirichlet and Neumann type boundary conditions ${ }^{5}$. A Robin problem is a mixed boundary value problem with a linear combination of Dirichlet and Neumann conditions, commonly called a Robin condition $^{6}$. Many analytical methods for solving the Robin boundary value problem for the Laplace equation $\nabla^{2} u=0$ in simply connected region are limited to special regions. For general shape region, we have to resort to numerical methods ${ }^{7}$. Alipour and Vali ${ }^{8}$ solved Volterra optimal control problems and applied the homotopy analysis method to solve the problem.

Recently, the interplay of Riemann-Hilbert (RH) problems and integral equations with the generalized Neumann kernel has been investigated for simply connected regions with smooth boundaries ${ }^{9}$ and for bounded and unbounded multiply connected regions ${ }^{10}$. Hamzah and Nasser ${ }^{11}$ and Hamzah et $\mathrm{al}^{12}$ have reformulated the interior Robin prob- lem as an $\mathrm{RH}$ problem and then utilized the results of Refs. 9, 10, but the exterior Robin problem is not treated. In Ref. 11 the method involves one integral equation with unknown constants $c_{1}, c_{2}$, a normalizing condition, and $\alpha, \beta$ limited to specific values. In Ref. 12 the method involves two integral equations without both normalizing condition and unknown constants $c_{1}, c_{2}$. In this paper, for bounded case, the approach is similar to Ref. 11, but with $\alpha, \beta$ now treated as functions. This paper presents a unified treatment of solving both interior and exterior Robin problems via $\mathrm{RH}$ problem and integral equations with the generalized Neumann kernel. In this paper, each Robin boundary value problem in both bounded and unbounded simply connected regions is reduced to an $\mathrm{RH}$ problem with normalizing conditions. However, in Ref. 13 each Robin boundary value problem in both bounded and unbounded multiply connected regions is reduced to two $\mathrm{RH}$ problems without normalizing conditions and the proof that the two integral equations are linearly independent is provided.

\section{NOTATION AND AUXILIARY MATERIAL}

Consider the interior and exterior Robin problems on an arbitrary simply connected region $\Omega$ with the boundary $\Gamma$. It consists of finding a harmonic 


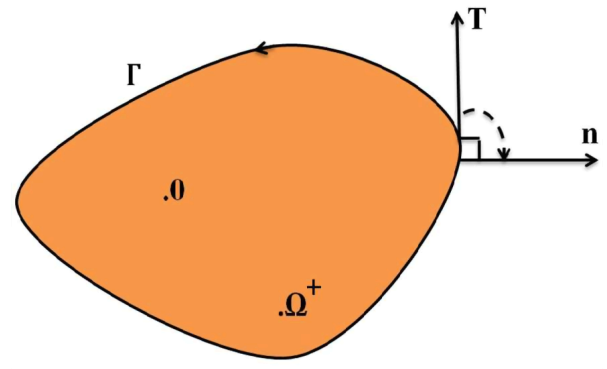

Fig. 1 A bounded simply connected region $\Omega^{+}$.

function $u$ continuous on $\Omega \cup \Gamma$ and satisfies the Robin boundary condition. We consider simply connected region $\Omega$ in the extended complex plane of the following two types.

(i) A bounded simply connected region $\Omega^{+}$with counter-clockwise orientation boundary $\Gamma$ which is a smooth Jordan curve (Fig. 1).

(ii) An unbounded simply connected region $\Omega^{-}$ with clockwise orientation boundary $\Gamma$ which is a smooth Jordan curve (Fig. 2).

The curve $\Gamma$ is parameterized by a $2 \pi$-periodic twice continuously differentiable complex function $\eta(t)$ with non-vanishing first derivative $\dot{\eta}(t) \neq 0$.

A Robin problem is a boundary value problem for determining a harmonic function $u(x, y)$ in $\Omega$ and continuous on $\Omega \cup \Gamma$ that satisfies the Robin boundary condition ${ }^{14}$

$$
\alpha(t) u(\eta(t))+\beta(t) \frac{\partial}{\partial \mathbf{n}} u(\eta(t))=l(t),
$$

where $\alpha(t) \neq 0, \beta(t) \neq 0$, and $l(t)$ are continuous, $\eta(t) \in \Gamma, \mathbf{n}$ is the exterior normal to $\Gamma, \partial u / \partial \mathbf{n}$ is the directional derivative of $u$ in the direction of $\mathbf{n}$. For unbounded $\Omega$, the function $u$ is also required to satisfy

$$
u(z) \rightarrow C \quad \text { as } \quad|z| \rightarrow \infty,
$$

with a constant $C$. In this paper, we focus on the case where $C=0$. The Robin problem is uniquely solvable under certain condition as given in the following theorem.

Theorem 1 If $\operatorname{sgn}(\alpha)=\operatorname{sgn}(\beta)$, then a harmonic function satisfying the Robin boundary condition (1) is unique ${ }^{15}$.

The function $u$ can be regarded as a real part of an analytic function $f=u+\mathrm{i} v$ in $\Omega$ that is continuous on $\Omega \cup \Gamma$. The unit tangent vector $\mathbf{T}$ of the boundary $\Gamma$ is obtained by $\mathbf{T}(t)=\dot{\eta}(t) /|\dot{\eta}(t)|$. The outward normal vector is a vector obtained by rotating the

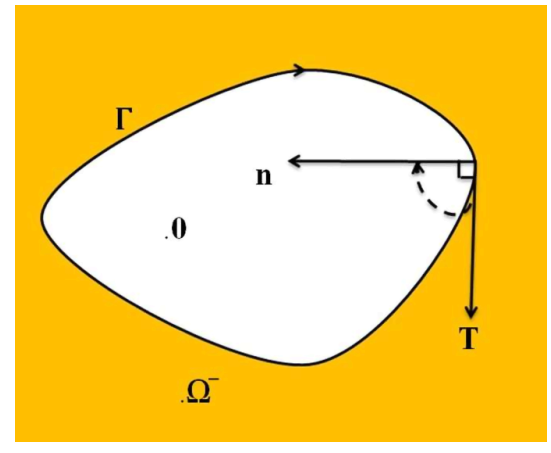

Fig. 2 An unbounded simply connected region $\Omega^{-}$.

unit tangent vector by $\pi / 2$ in a clockwise direction, i.e., $\mathbf{n}=-\mathrm{iT}$, which gives

$$
\mathbf{n}(\eta(t))=\mathrm{e}^{\mathrm{i} \theta(\eta(t))}=-\mathrm{i} \mathbf{T}(\eta(t)),
$$

where $\theta(\eta(t))$ is the angle between the exterior normal vector $\mathbf{n}$ and and positive real axis (see Figs. 1 and 2).

Let $A(t)$ be a continuous differentiable $2 \pi$ periodic function with $A(t) \neq 0$. Define two real kernels ${ }^{9}$ by

$$
\begin{array}{ll}
M(s, t)=\frac{1}{\pi} \operatorname{Re}\left[\frac{A(s)}{A(t)} \frac{\dot{\eta}(t)}{\eta(t)-\eta(s)}\right], & s \neq t, \\
N(s, t)=\frac{1}{\pi} \operatorname{Im}\left[\frac{A(s)}{A(t)} \frac{\dot{\eta}(t)}{\eta(t)-\eta(s)}\right], & s \neq t .
\end{array}
$$

The kernel $N(s, t)$ is called the generalized Neumann kernel formed with $A(t)$ and $\eta(t)$. When $A(t)=1$, it reduces to the classical Neumann kernel,

$$
N(s, t)=\frac{1}{\pi} \operatorname{Im}\left[\frac{\dot{\eta}(t)}{\eta(t)-\eta(s)}\right], \quad s \neq t .
$$

The generalized Neumann kernel is continuous at $t=s$ with

$$
N(t, t)=\frac{1}{\pi} \operatorname{Im}\left[\frac{1}{2} \frac{\ddot{\eta}(t)}{\dot{\eta}(t)}-\frac{\dot{A}(t)}{A(t)}\right], \quad t=s .
$$

The kernel $M(s, t)$ has the representation

$$
M(s, t)=-\frac{1}{2 \pi} \cot \frac{s-t}{2}+M_{1}(s, t), \quad s \neq t,
$$

with the continuous kernel $M_{1}$ having the diagonal the values

$$
M_{1}(t, t)=\frac{1}{\pi} \operatorname{Re}\left[\frac{1}{2} \frac{\ddot{\eta}(t)}{\dot{\eta}(t)}-\frac{\dot{A}(t)}{A(t)}\right] .
$$


Let $\mathbf{N}$ and $\mathbf{M}_{1}$ be the Fredholm integral operators associated with the continuous kernels $N$ and $M_{1}$, respectively,

$$
\begin{aligned}
(\mathbf{N} \mu)(s) & =\int_{0}^{2 \pi} N(s, t) \mu(t) \mathrm{d} t \\
\left(\mathbf{M}_{1} \mu\right)(s) & =\int_{0}^{2 \pi} M_{1}(s, t) \mu(t) \mathrm{d} t .
\end{aligned}
$$

The adjoint of the operator $\mathbf{N}$ is defined by

$$
\left(\mathbf{N}^{*} \mu\right)(s)=\int_{0}^{2 \pi} N^{*}(s, t) \mu(t) \mathrm{d} t,
$$

where $N^{*}(s, t)=N(t, s)$. Let $\mathbf{M}$ and $\mathbf{K}$ be the singular integral operators

$$
\begin{aligned}
& (\mathbf{M} \mu)(s)=\int_{0}^{2 \pi} M(s, t) \mu(t) \mathrm{d} t \\
& (\mathrm{~K} \mu)(s)=\frac{1}{2 \pi} \int_{0}^{2 \pi} \cot \frac{s-t}{2} \mathrm{~d} t .
\end{aligned}
$$

The integrals (11) and (12) are principal value integrals. The operator $\mathbf{K}$ is known as the conjugation operator. It is also known as the Hilbert transform ${ }^{9}$. It follows from (6) that $\mathbf{M}=\mathbf{M}_{1}-\mathbf{K}$.

Let $\gamma(t)$ be a real Hölder continuously periodic function with period $2 \pi$. The RH problem consists of finding an analytic function $f$ in $\Omega$ that is continuous in its closure $\bar{\Omega}$ and has boundary values $f(\eta(t))$ satisfying

$$
\operatorname{Re}[A(t) f(\eta(t))]=\gamma(t) .
$$

For unbounded $\Omega$, due to $u(z) \rightarrow 0$ as $|z| \rightarrow \infty$, the function $f$ is also required to satisfy $f(\infty)=$ 0 . If $\operatorname{Re}[A(t) f(\eta(t))]=0$ we have a homogeneous boundary condition.

Theorem 2 If $g$ is a solution of the RH problem (13) with boundary values ${ }^{9}$

$$
A g=\gamma+\mathrm{i} \mu,
$$

then the imaginary part $\mu$ satisfies the integral equation

$$
\mu-\mathbf{N} \mu=-\mathbf{M} \gamma .
$$

The solvability of boundary integral equations with the generalized Neumann kernel is determined by the index ${ }^{9}$ of the function $A(t)$, defined as the winding number of $A(t)$ with respected to 0 . The index of $A(t)$ is denoted by $\kappa_{A}$, which is ${ }^{5,9}$

$$
\kappa_{A}=\left.\frac{1}{2 \pi} \Delta \arg A(t)\right|_{0} ^{2 \pi},
$$

or, if $A(t)$ is differentiable,

$$
\kappa_{A}=\frac{1}{2 \pi \mathrm{i}} \int_{0}^{2 \pi} \frac{\dot{A}(t)}{A(t)} \mathrm{d} t .
$$

Since $\Gamma$ is closed and $A(t)$ is a non-vanishing continuous function on $\Gamma$, the index $\kappa_{A}$ is an integer. If $\kappa_{A}>0$, then it can be shown that (15) has a unique solution $^{9,16}$. Define the integral operator ${ }^{17} \mathbf{J}$ by

$$
\mathbf{J} \mu=\frac{1}{2 \pi} \int_{0}^{2 \pi} \mu(t) \mathrm{d} t,
$$

which is a constant.

Theorem 3 Let $h$ be a real constant function such that

$$
A f=\gamma+h+\mathrm{i} \mu
$$

are the boundary values of the analytic function $f(z)$ in $\Omega$. Then the function $h$ is given by

$$
h=(\gamma, \phi),
$$

where $\phi$ is the unique solution of the integral equation

$$
\left(\mathbf{I}+\mathbf{N}^{*}+\mathbf{J}\right) \phi= \pm 1,
$$

where + sign for bounded region and - sign for unbounded region ${ }^{17}$.

Theorem 4 (Cauchy Integral Formula) ${ }^{5}$ Let $f$ be analytic function everywhere in $\Omega$ and on a simple closed contour $\Gamma$. The Cauchy integral formulae for $\Omega^{+}$and $\Omega^{-}$, respectively, are given by

$$
\begin{gathered}
\frac{1}{2 \pi \mathrm{i}} \int_{\Gamma} \frac{f(\eta)}{\eta-z} \mathrm{~d} \eta= \begin{cases}f(z), & z \in \Omega^{+}, \\
0, & z \in \Omega^{-},\end{cases} \\
\frac{1}{2 \pi \mathrm{i}} \int_{\Gamma} \frac{f(\eta)}{\eta-z} \mathrm{~d} \eta= \begin{cases}f(z)-f(\infty), & z \in \Omega^{-}, \\
-f(\infty), & z \in \Omega^{+} .\end{cases}
\end{gathered}
$$

\section{REDUCTION OF ROBIN BOUNDARY VALUE PROBLEM TO RH-PROBLEM}

We consider the Robin problem (1) either interior or exterior. The unit exterior normal vector is given by

$$
\begin{aligned}
\mathbf{n}(\eta(t)) & =-\mathrm{i} \mathbf{T}(\eta(t))=-\mathrm{i} \frac{\dot{\eta}}{|\dot{\eta}|}=\mathrm{e}^{\mathrm{i} \theta(\eta(t))} \\
& =\cos \theta(\eta(t))+\mathrm{i} \sin \theta(\eta(t)) .
\end{aligned}
$$

Then

$$
\begin{aligned}
\frac{\partial u}{\partial \mathbf{n}} & =\cos \theta(\eta(t)) \frac{\partial u}{\partial x}+\sin \theta(\eta(t)) \frac{\partial u}{\partial y} \\
& =\operatorname{Re}\left[\mathrm{e}^{\mathrm{i} \theta(\eta(t))}\left(\frac{\partial u}{\partial x}-\mathrm{i} \frac{\partial u}{\partial y}\right)\right] .
\end{aligned}
$$


Since $f(z)=u+\mathrm{i} v$, then by the Cauchy-Riemann equations we have, $f^{\prime}(z)=u_{x}-\mathrm{i} u_{y}$. Thus

$$
\frac{\partial u(\eta(t))}{\partial \mathbf{n}}=\operatorname{Re}\left[-\mathrm{i} \frac{\dot{\eta}(t)}{|\dot{\eta}(t)|} f^{\prime}(\eta(t))\right] .
$$

Substituting into the Robin condition (1), we obtain

$$
\alpha(t) \operatorname{Re}[f(\eta(t))]-\beta(t) \operatorname{Re}\left[\frac{\mathrm{i} \dot{\eta}(t) f^{\prime}(\eta(t))}{|\dot{\eta}(t)|}\right]=l(t) .
$$

It is sometimes assumed that $\eta(t)$ is the arc length parameterization of the boundary $\Gamma$, which implies $|\dot{\eta}(t)|=1$. This assumption, although convenient for theoretical work, but in numerical aspect, it introduces an additional sources of error. If $\Omega$ is a unit disc, (24) yields the same result as in Petrila ${ }^{14}$. Multiply both sides by $|\dot{\eta}(t)|$ we obtain

$$
\begin{array}{r}
\operatorname{Re}\left[\alpha(t)|\dot{\eta}(t)| f(\eta(t))-\mathrm{i} \beta(t) \dot{\eta}(t) f^{\prime}(\eta(t))\right] \\
=l(t)|\dot{\eta}(t)|,
\end{array}
$$

or, equivalently,

$$
\begin{array}{r}
\operatorname{Re}\left[-\mathrm{i} \beta(t)\left\{\frac{\mathrm{d}}{\mathrm{d} t}(f(\eta(t)))+\mathrm{i} c(t)\right.\right. \\
f(\eta(t))\}] \\
=l(t)|\dot{\eta}(t)|,
\end{array}
$$

where $c(t)=(\alpha(t) / \beta(t))|\dot{\eta}(t)|$. Eq. (26) may also be written as

$$
\begin{aligned}
-\mathrm{i} \beta(t)\left[\frac{\mathrm{d}}{\mathrm{d} t}(f(\eta(t)))+\mathrm{i} c(t) f(\eta(t))\right] \\
=l(t)|\dot{\eta}(t)|+\mathrm{i} \mu(t),
\end{aligned}
$$

where $\mu(t)$ is an unknown function. By means of integrating factor, we obtain

$$
\begin{array}{r}
-\mathrm{i} \beta(t) A(t)\left[\frac{\mathrm{d}}{\mathrm{d} t}(f(\eta(t)))+\mathrm{i} c(t) f(\eta(t))\right] \\
=l(t)|\dot{\eta}(t)| \mathrm{e}^{\mathrm{i} \kappa(t)}+\mathrm{i} \mu(t) \mathrm{e}^{\mathrm{i} \kappa(t)},
\end{array}
$$

where $A(t)=\mathrm{e}^{\mathrm{i} \kappa(t)}$ when $\kappa(t)=\int_{0}^{t} c(\tau) \mathrm{d} \tau$. Then (28) becomes

$$
\frac{\mathrm{d}}{\mathrm{d} t}[-\mathrm{i} A(t) f(\eta(t))]=\frac{\mathrm{e}^{\mathrm{i} \kappa(t)}}{\beta(t)}(l(t)|\dot{\eta}(t)|+\mathrm{i} \mu(t)) .
$$

Letting $g=-\mathrm{i} f$, which is analytic on $\Omega$, we obtain

$$
\begin{gathered}
A(t) g(\eta(t))=\int_{0}^{t} \frac{l(\tau)|\dot{\eta}(\tau)| \cos \kappa(\tau)}{\beta(\tau)} \mathrm{d} \tau \\
+\mathrm{i} \int_{0}^{t} \frac{l(\tau)|\dot{\eta}(\tau)| \sin \kappa(\tau)}{\beta(\tau)} \mathrm{d} \tau
\end{gathered}
$$

$$
\begin{gathered}
+\mathrm{i} \int_{0}^{t} \frac{\mu(\tau) \cos \kappa(\tau)}{\beta(\tau)} \mathrm{d} \tau \\
\quad-\int_{0}^{t} \frac{\mu(\tau) \sin \kappa(\tau)}{\beta(\tau)} \mathrm{d} \tau+c_{1}+\mathrm{i} c_{2} \\
=\gamma_{1}(t)+\mathrm{i} \gamma_{2}(t)+\mathrm{i} \mu_{1}(t)-\mu_{2}(t)+c_{1}+\mathrm{i} c_{2},
\end{gathered}
$$

where $c_{1}$ and $c_{2}$ are unknown real constants, and

$$
\begin{aligned}
& \gamma_{1}(t)=\int_{0}^{t} \frac{l(\tau)|\dot{\eta}(\tau)| \cos \kappa(\tau)}{\beta(\tau)} \mathrm{d} \tau, \\
& \gamma_{2}(t)=\int_{0}^{t} \frac{l(\tau)|\dot{\eta}(\tau)| \sin \kappa(\tau)}{\beta(\tau)} \mathrm{d} \tau
\end{aligned}
$$

are known functions, and

$$
\begin{aligned}
& \mu_{1}(t)=\int_{0}^{t} \frac{\mu(\tau) \cos \kappa(\tau)}{\beta(\tau)} \mathrm{d} \tau, \\
& \mu_{2}(t)=\int_{0}^{t} \frac{\mu(\tau) \sin \kappa(\tau)}{\beta(\tau)} \mathrm{d} \tau
\end{aligned}
$$

are unknown functions. Note that (30) is an $\mathrm{RH}$ problem in the form of (14). The function $A(t)=$ $\mathrm{e}^{\mathrm{i} \kappa(t)}$ in general is not periodic. $A(t)$ must be periodic so we can calculate the index and apply Theorem 2. The function $A(t)$ is periodic if we assume $\kappa(2 \pi)-\kappa(0)=2 \pi$ such that the index $\kappa_{A}=$ 1 . With this restriction, we are solving a special case of the Robin problem for which $\alpha(t)$ and $\beta(t)$ satisfy the condition $\kappa(2 \pi)-\kappa(0)=2 \pi$. Thus Theorem 2 implies that (30) can be reformulated as

$(\mathbf{I}-\mathbf{N})\left(\gamma_{2}(s)+\mu_{1}(s)+c_{2}\right)=-\mathbf{M}\left(\gamma_{1}(s)-\mu_{2}(s)+c_{1}\right)$,

or in another form

$$
\begin{array}{r}
(\mathbf{I}-\mathbf{N}) \mu_{1}(s)-\mathbf{M} \mu_{2}(s)+c_{1} \mathbf{M}+c_{2}(\mathbf{I}-\mathbf{N}) \\
=-(\mathbf{I}-\mathbf{N}) \gamma_{2}(s)-\mathbf{M} \gamma_{1}(s),
\end{array}
$$

which is equivalent to

$$
\begin{gathered}
\mu_{1}(s)-\int_{0}^{2 \pi} N(s, t) \mu_{1}(t) \mathrm{d} t-\int_{0}^{2 \pi} M(s, t) \mu_{2}(t) \mathrm{d} t \\
+\int_{0}^{2 \pi} K(s, t) \mu_{2}(t) \mathrm{d} t+c_{1} \int_{0}^{2 \pi} M_{1}(s, t) \mathrm{d} t \\
-c_{1} \int_{0}^{2 \pi} K(s, t) \mathrm{d} t+c_{2}-c_{2} \int_{0}^{2 \pi} N(s, t) \mathrm{d} t \\
=-\gamma_{2}(s)+\int_{0}^{2 \pi} N(s, t) \gamma_{2}(t) \mathrm{d} t
\end{gathered}
$$




$$
-\int_{0}^{2 \pi} M(s, t) \gamma_{1}(t) \mathrm{d} t+\int_{0}^{2 \pi} K(s, t) \gamma_{1}(t) \mathrm{d} t .
$$

This is an integral equation in two unknown real functions $\mu_{1}$ and $\mu_{2}$ and two unknown constants $c_{1}$ and $c_{2}$. The integral equation (37) has an infinite number of solutions. To obtain a unique solution, sufficient conditions need to be determined. By the definitions of the function $\mu_{1}$ and $\mu_{2}$, we have a condition in the form of a differential equation

$$
\sin (\kappa(t)) \mu_{1}^{\prime}(t)-\cos (\kappa(t)) \mu_{2}^{\prime}(t)=0,
$$

with conditions

$$
\mu_{1}(0)=\mu_{2}(0)=0 .
$$

The above constructions for interior Robin problem ${ }^{11}$, is also valid for the exterior Robin problem. For the interior Robin problem ${ }^{9}$ we have $\operatorname{Im}[f(0)]=$ 0 , which implies that $\operatorname{Re}[g(0)]=0$. Let

$$
\begin{aligned}
& \psi=\gamma_{1}(t)-\mu_{2}(t)+c_{1}, \\
& \varphi=\gamma_{2}(t)+\mu_{1}(t)+c_{2} .
\end{aligned}
$$

The Cauchy integral formula Theorem 4 yields

$$
g(0)=\frac{1}{2 \pi \mathrm{i}} \int_{0}^{2 \pi} \frac{(\psi+\mathrm{i} \varphi) \dot{\eta}(t)}{A(t) \eta(t)} \mathrm{d} t,
$$

which gives

$$
\int_{0}^{2 \pi}\left(\psi \operatorname{Im}\left[\frac{\dot{\eta}(t)}{A(t) \eta(t)}\right]+\varphi \operatorname{Re}\left[\frac{\dot{\eta}(t)}{A(t) \eta(t)}\right]\right) \mathrm{d} t=0 .
$$

Hence, we obtain another condition in the form

$$
\begin{aligned}
& \int_{0}^{2 \pi} \operatorname{Re}[w(t)] \mu_{1}(t) \mathrm{d} t-\int_{0}^{2 \pi} \operatorname{Im}[w(t)] \mu_{2}(t) \mathrm{d} t \\
& \quad+c_{1} \int_{0}^{2 \pi} \operatorname{Im}[w(t)] \mathrm{d} t+c_{2} \int_{0}^{2 \pi} \operatorname{Re}[w(t)] \mathrm{d} t \\
& =-\int_{0}^{2 \pi} \operatorname{Re}[w(t)] \gamma_{2}(t) \mathrm{d} t-\int_{0}^{2 \pi} \operatorname{Im}[w(t)] \gamma_{1}(t) \mathrm{d} t,
\end{aligned}
$$

where $w(t)=\dot{\eta}(t) / A(t) \eta(t)$. From (30), we have $A(t) g(\eta(t))=\gamma_{1}(t)-\mu_{2}(t)+c_{1}+\mathrm{i}\left(\gamma_{2}(t)+\mu_{1}(t)+c_{2}\right)$.

Hence, the following $\mathrm{RH}$ problems are solvable,

$$
\operatorname{Re}[A(t) g(\eta(t))]=\gamma_{1}(t)-\mu_{2}(t)+c_{1}
$$

and

$$
\operatorname{Im}[A(t) g(\eta(t))]=\gamma_{2}(t)+\mu_{1}(t)+c_{2} .
$$

Thus the functions $\gamma_{1}(t)-\mu_{2}(t)+c_{1}$ and $\gamma_{2}(t)+$ $\mu_{1}(t)+c_{2}$ are orthogonal with the eigenfunction $\phi$ of the adjoint operator $\mathbf{N}^{*}$ corresponding to the eigenvalue $\lambda=-1$. Using the normalization ${ }^{9} \mathrm{~J} \phi=$ 1 , and since we have ${ }^{17}\left(\mathbf{I}+\mathbf{N}^{*}\right) \phi=0$, (19) becomes

$$
\left(\mathbf{I}+\mathbf{N}^{*}+\mathbf{J}\right) \phi=1 .
$$

Thus for the interior Robin problem, the function $\mu_{1}$ and constant $c_{2}$ satisfy

$$
\begin{aligned}
& \frac{1}{2 \pi} \int_{0}^{2 \pi}\left(\gamma_{2}(t)+\mu_{1}(t)+c_{2}\right) \phi(t) \mathrm{d} t=0 \\
& \frac{1}{2 \pi} \int_{0}^{2 \pi} \phi(t) \mu_{1}(t) \mathrm{d} t+c_{2}=\frac{-1}{2 \pi} \int_{0}^{2 \pi} \phi(t) \gamma_{2} \mathrm{~d} t
\end{aligned}
$$

while the function $\mu_{2}$, and constant $c_{1}$ satisfy

$$
\begin{aligned}
& \frac{1}{2 \pi} \int_{0}^{2 \pi}\left(\gamma_{1}(t)-\mu_{2}(t)+c_{1}\right) \phi(t) \mathrm{d} t=0 \\
& \frac{-1}{2 \pi} \int_{0}^{2 \pi} \phi(t) \mu_{2}(t) \mathrm{d} t+c_{1}=\frac{-1}{2 \pi} \int_{0}^{2 \pi} \phi(t) \gamma_{1} \mathrm{~d} t .
\end{aligned}
$$

The conditions (42), (44) and (46) are also given in Ref. 11 for the interior Robin problem. But a different set of conditions are required for the exterior Robin problem. For the exterior Robin problem, we have $f(\infty)=0$, which implies $g(\infty)=0$. By using the Cauchy integral formula for unbounded region $\Omega$ Theorem 4, we have

$$
\frac{1}{2 \pi \mathrm{i}} \int_{\Gamma} \frac{g(\eta)}{\eta-z} \mathrm{~d} \eta= \begin{cases}g(z)-g(\infty), & z \in \Omega^{-}, \\ -g(\infty), & z \in \Omega^{+} .\end{cases}
$$

Choosing $z=0$ outside $\Omega^{-}$gives

$$
\frac{1}{2 \pi \mathrm{i}} \int_{\Gamma} \frac{g(\eta)}{\eta} \mathrm{d} \eta=-g(\infty)=0 .
$$

Applying (30), we obtain

$$
\frac{1}{2 \pi \mathrm{i}} \int_{\Gamma} \frac{\psi+\mathrm{i} \varphi}{A(t)} \frac{\mathrm{d} \eta(t)}{\eta(t)}=0,
$$

which implies

$$
\begin{aligned}
& \int_{0}^{2 \pi}\left(\psi \operatorname{Im} \frac{\dot{\eta}(t)}{A(t) \eta(t)}+\varphi \operatorname{Re} \frac{\dot{\eta}(t)}{A(t) \eta(t)}\right) \mathrm{d} t \\
& -\mathrm{i} \int_{0}^{2 \pi}\left(\psi \operatorname{Re} \frac{\dot{\eta}(t)}{A(t) \eta(t)}-\varphi \operatorname{Im} \frac{\dot{\eta}(t)}{A(t) \eta(t)}\right) \mathrm{d} t=0 .
\end{aligned}
$$


The real and imaginary parts of (50), yield

$$
\begin{aligned}
& \int_{0}^{2 \pi}\left(\psi \operatorname{Im} \frac{\dot{\eta}(t)}{A(t) \eta(t)}+\varphi \operatorname{Re} \frac{\dot{\eta}(t)}{A(t) \eta(t)}\right) \mathrm{d} t=0, \\
& \int_{0}^{2 \pi}\left(\psi \operatorname{Re} \frac{\dot{\eta}(t)}{A(t) \eta(t)}-\varphi \operatorname{Im} \frac{\dot{\eta}(t)}{A(t) \eta(t)}\right) \mathrm{d} t=0,
\end{aligned}
$$

which gives rise to the conditions

$$
\begin{aligned}
& \int_{0}^{2 \pi} \operatorname{Re}[w(t)] \mu_{1}(t) \mathrm{d} t-\int_{0}^{2 \pi} \operatorname{Im}[w(t)] \mu_{2}(t) \mathrm{d} t \\
& \quad+c_{1} \int_{0}^{2 \pi} \operatorname{Im}[w(t)] \mathrm{d} t+c_{2} \int_{0}^{2 \pi} \operatorname{Re}[w(t)] \mathrm{d} t \\
& =-\int_{0}^{2 \pi} \operatorname{Re}[w(t)] \gamma_{2}(t) \mathrm{d} t-\int_{0}^{2 \pi} \operatorname{Im}[w(t)] \gamma_{1}(t) \mathrm{d} t,
\end{aligned}
$$

and

$$
\begin{gathered}
-\int_{0}^{2 \pi} \operatorname{Im}[w(t)] \mu_{1}(t) \mathrm{d} t-\int_{0}^{2 \pi} \operatorname{Re}[w(t)] \mu_{2}(t) \mathrm{d} t \\
+c_{1} \int_{0}^{2 \pi} \operatorname{Re}[w(t)] \mathrm{d} t-c_{2} \int_{0}^{2 \pi} \operatorname{Im}[w(t)] \mathrm{d} t \\
=\int_{0}^{2 \pi} \operatorname{Im}[w(t)] \gamma_{2}(t) \mathrm{d} t-\int_{0}^{2 \pi} \operatorname{Re}[w(t)] \gamma_{1}(t) \mathrm{d} t
\end{gathered}
$$

where $w(t)=\dot{\eta}(t) / A(t) \eta(t)$.

The functions $\gamma_{1}(t)-\mu_{2}(t)+c_{1}$ and $\gamma_{2}(t)+$ $\mu_{1}(t)+c_{2}$ are orthogonal with the eigenfunction $\phi$ of the adjoint operator $\mathbf{N}^{*}$ corresponding to the eigenvalue $\lambda=-1$. Using the normalization ${ }^{9} \mathbf{J} \phi=$ -1 and condition ${ }^{17}\left(\mathbf{I}+\mathbf{N}^{*}\right) \phi=0$, (19) becomes

$$
\left(\mathbf{I}+\mathbf{N}^{*}+\mathbf{J}\right) \phi=-1 .
$$

Then for the exterior Robin problem, the function $\mu_{1}$ and constant $c_{2}$ satisfy

$$
\begin{aligned}
& \frac{1}{2 \pi} \int_{0}^{2 \pi}\left(\gamma_{2}(t)+\mu_{1}(t)+c_{2}\right) \phi(t) \mathrm{d} t=0 \\
& \frac{1}{2 \pi} \int_{0}^{2 \pi} \phi(t) \mu_{1}(t) \mathrm{d} t-c_{2}=\frac{-1}{2 \pi} \int_{0}^{2 \pi} \phi(t) \gamma_{2} \mathrm{~d} t
\end{aligned}
$$

while the function $\mu_{2}$ and constant $c_{1}$ satisfy

$$
\frac{1}{2 \pi} \int_{0}^{2 \pi}\left(\gamma_{1}(t)-\mu_{2}(t)+c_{1}\right) \phi(t) \mathrm{d} t=0,
$$

$$
\frac{-1}{2 \pi} \int_{0}^{2 \pi} \phi(t) \mu_{2}(t) \mathrm{d} t-c_{1}=\frac{-1}{2 \pi} \int_{0}^{2 \pi} \phi(t) \gamma_{1} \mathrm{~d} t
$$

\section{NUMERICAL IMPLEMENTATION}

Since the functions $A(t)$ and $\eta(t)$ are $2 \pi$-periodic, the integral equation (36) can be best discretized on an equidistant grid by the Nyström method with trapezoidal rule using $n$ equidistant nodes ${ }^{18}$. The integral involving the singular kernel $K(s, t)$ is discretized using the Wittich method ${ }^{19}$. Define the $n$ equidistant collocation points $t_{i}$ by $t_{i}=2 \pi(i-1) / n$, for $i=1,2,3, \ldots, n$.

Discretizing the integral equation (37), we obtain the linear system

$$
\begin{gathered}
\mu_{1}\left(t_{i}\right)-\frac{2 \pi}{n} \sum_{j=1}^{n} N\left(t_{i}, t_{j}\right) \mu_{1}\left(t_{j}\right) \\
-\frac{2 \pi}{n} \sum_{j=1}^{n} M\left(t_{i}, t_{j}\right) \mu_{2}\left(t_{j}\right)+\sum_{j=1}^{n} K\left(t_{i}, t_{j}\right) \mu_{2}\left(t_{j}\right) \\
+c_{1} \frac{2 \pi}{n} \sum_{j=1}^{n} M\left(t_{i}, t_{j}\right)-c_{1} \sum_{j=1} n K\left(t_{i}, t_{j}\right) \\
\quad+c_{2}-c_{2} \frac{2 \pi}{n} \sum_{j=1}^{n} N\left(t_{i}, t_{j}\right) \\
=-\gamma_{2}\left(t_{i}\right)+\frac{2 \pi}{n} \sum_{j=1}^{n} N\left(t_{i}, t_{j}\right) \gamma_{2}\left(t_{j}\right) \\
-\frac{2 \pi}{n} \sum_{j=1}^{n} M\left(t_{i}, t_{j}\right) \gamma_{1}\left(t_{j}\right)+\sum_{j=1}^{n} K\left(t_{i}, t_{j}\right) \gamma_{1}\left(t_{j}\right),
\end{gathered}
$$

where

$$
\begin{aligned}
K\left(t_{i}, t_{j}\right) & = \begin{cases}0, & j-i \text { is even, } \\
\frac{2}{n} \cot \frac{(i-j) \pi}{n}, & j-i \text { is odd, }\end{cases} \\
N\left(t_{i}, t_{j}\right) & =\left\{\begin{array}{cl}
\frac{1}{\pi} \operatorname{Im}\left[\frac{A\left(t_{i}\right)}{A\left(t_{j}\right)} \frac{\dot{\eta}\left(t_{j}\right)}{\eta\left(t_{j}\right)-\eta\left(t_{i}\right)}\right], & t_{i} \neq t_{j}, \\
\frac{1}{\pi} \operatorname{Im}\left[\frac{1}{2} \frac{\dot{\eta}(t)}{\dot{\eta}(t)}-\frac{\dot{A}(t)}{A(t)}\right], & t_{i}=t_{j},
\end{array}\right. \\
M_{1}\left(t_{i}, t_{j}\right) & =\left\{\begin{array}{cc}
\frac{1}{\pi} \operatorname{Re}\left[\frac{A\left(t_{i}\right)}{A\left(t_{j}\right)} \frac{\dot{\eta}\left(t_{j}\right)}{\eta\left(t_{j}\right)-\eta\left(t_{i}\right)}\right], & \\
+\frac{1}{2 \pi} \cot \frac{t_{i}-t_{j}}{2}, & t_{i} \neq t_{j}, \\
\frac{1}{\pi} \operatorname{Re}\left[\frac{1}{2} \frac{\ddot{\eta}(t)}{\dot{\eta}(t)}-\frac{\dot{A}(t)}{A(t)}\right], & t_{i}=t_{j} .
\end{array}\right.
\end{aligned}
$$

Hence we obtain $n$ equations in $2 n+2$ variables

$$
\mu_{1}\left(t_{1}\right), \ldots, \mu_{1}\left(t_{n}\right), \mu_{2}\left(t_{1}\right), \ldots, \mu_{2}\left(t_{n}\right), c_{1}, c_{2} .
$$

The condition (38) will be discretized using a fivepoint central difference method to obtain $n$ equa- 
tions ${ }^{20}$. For $i=1$, we obtain

$$
\begin{aligned}
u_{1}^{\prime}\left(t_{1}\right) \approx u_{11}^{\prime}= & -25 \mu_{1}\left(t_{1}\right)+48 \mu_{1}\left(t_{2}\right)-36 \mu_{1}\left(t_{3}\right) \\
& +16 \mu_{1}\left(t_{4}\right)-3 \mu_{1}\left(t_{5}\right) . \\
u_{2}^{\prime}\left(t_{1}\right) \approx u_{21}^{\prime}= & -25 \mu_{2}\left(t_{1}\right)+48 \mu_{2}\left(t_{2}\right)-36 \mu_{2}\left(t_{3}\right) \\
& +16 \mu_{2}\left(t_{4}\right)-3 \mu_{2}\left(t_{5}\right) .
\end{aligned}
$$

For $i=2$, we have

$$
\begin{aligned}
u_{1}^{\prime}\left(t_{2}\right) \approx u_{12}^{\prime}= & -3 \mu_{1}\left(t_{1}\right)-10 \mu_{1}\left(t_{2}\right)+18 \mu_{1}\left(t_{3}\right) \\
& -6 \mu_{1}\left(t_{4}\right)+\mu_{1}\left(t_{5}\right) . \\
u_{2}^{\prime}\left(t_{2}\right) \approx u_{22}^{\prime}= & -3 \mu_{2}\left(t_{1}\right)-10 \mu_{2}\left(t_{2}\right)+18 \mu_{2}\left(t_{3}\right) \\
& -6 \mu_{2}\left(t_{4}\right)+\mu_{2}\left(t_{5}\right) .
\end{aligned}
$$

For $i=3, \ldots, n-2$, we have

$$
\begin{aligned}
u_{1}^{\prime}\left(t_{i}\right) \approx u_{1 i}^{\prime}= & \mu_{1}\left(t_{i-2}\right)-8 \mu_{1}\left(t_{i-1}\right)+8 \mu_{1}\left(t_{i+1}\right) \\
& -\mu_{1}\left(t_{i+2}\right) . \\
u_{2}^{\prime}\left(t_{i}\right) \approx u_{2 i}^{\prime}= & \mu_{2}\left(t_{i-2}\right)-8 \mu_{2}\left(t_{i-1}\right)+8 \mu_{2}\left(t_{i+1}\right) \\
& -\mu_{2}\left(t_{i+2}\right) .
\end{aligned}
$$

For $i=n-1$, we have

$$
\begin{gathered}
u_{1}^{\prime}\left(t_{n-1}\right) \approx u_{1(n-1)}^{\prime}=-\mu_{1}\left(t_{n-4}\right)+6 \mu_{1}\left(t_{n-3}\right) \\
-18 \mu_{1}\left(t_{n-2}\right)+10 \mu_{1}\left(t_{n-1}\right)+3 \mu_{1}\left(t_{n}\right) . \\
u_{2}^{\prime}\left(t_{n-1}\right) \approx u_{2(n-1)}^{\prime}=-\mu_{2}\left(t_{n-4}\right)+6 \mu_{2}\left(t_{n-3}\right) \\
-18 \mu_{2}\left(t_{n-2}\right)+10 \mu_{2}\left(t_{n-1}\right)+3 \mu_{2}\left(t_{n}\right) .
\end{gathered}
$$

For $i=n$, we have

$$
\begin{aligned}
u_{1}^{\prime}\left(t_{n}\right) & \approx u_{1 n}^{\prime}=3 \mu_{1}\left(t_{n-4}\right)-16 \mu_{1}\left(t_{n-3}\right) \\
& +36 \mu_{1}\left(t_{n-2}\right)-48 \mu_{1}\left(t_{n-1}\right)+25 \mu_{1}\left(t_{n}\right) . \\
u_{2}^{\prime}\left(t_{n}\right) \approx & u_{2 n}^{\prime}=3 \mu_{2}\left(t_{n-4}\right)-16 \mu_{2}\left(t_{n-3}\right) \\
& +36 \mu_{2}\left(t_{n-2}\right)-48 \mu_{2}\left(t_{n-1}\right)+25 \mu_{2}\left(t_{n}\right) .
\end{aligned}
$$

We now have $2 n$ equations in $2 n+2$ variables in (60). Combining with the two conditions (39) gives a $(2 n+2) \times(2 n+2)$ linear system. Discretizing the condition (42) gives

$$
\begin{aligned}
& \sum_{j=1}^{n} \operatorname{Re}\left[w\left(t_{j}\right)\right] \mu_{1}\left(t_{j}\right)-\sum_{j=1}^{n} \operatorname{Im}\left[w\left(t_{j}\right)\right] \mu_{2}\left(t_{j}\right) \\
& +c_{1} \sum_{j=1}^{n} \operatorname{Im}\left[w\left(t_{j}\right)\right]+c_{2} \sum_{j=1}^{n} \operatorname{Re}\left[w\left(t_{j}\right)\right] \\
& =-\sum_{j=1}^{n} \operatorname{Re}\left[w\left(t_{j}\right)\right] \gamma_{2}\left(t_{j}\right)-\sum_{j=1}^{n} \operatorname{Im}\left[w\left(t_{j}\right)\right] \gamma_{1}\left(t_{j}\right),
\end{aligned}
$$

where $w(t)=\dot{\eta}(t) / A(t) \eta(t)$. Discretizing the conditions (44) and (46) gives

$$
\begin{gathered}
\frac{1}{n} \sum_{j=1}^{n} \phi\left(t_{j}\right) \mu_{1}\left(t_{j}\right)+c_{2}=\frac{-1}{n} \sum_{j=1}^{n} \phi\left(t_{j}\right) \gamma_{2}\left(t_{j}\right), \\
\frac{-1}{n} \sum_{j=1}^{n} \phi\left(t_{j}\right) \mu_{2}\left(t_{j}\right)+c_{1}=\frac{-1}{n} \sum_{j=1}^{n} \phi\left(t_{j}\right) \gamma_{1}\left(t_{j}\right) .
\end{gathered}
$$

Hence, for the Robin problem in bounded simply connected region we obtain $2 n \times(2 n+2)$ linear system, including the two conditions (39) gives a $(2 n+2) \times(2 n+2)$ linear system. Adding the equation obtained from (61) makes a $(2 n+3) \times(2 n+2)$ linear system. Finally, the two more equations from (62) and (63) are added to give a $(2 n+5) \times(2 n+2)$ linear system. For the unbounded Robin problem with conditions (53) and (54), we obtain

$$
\begin{aligned}
\sum_{j=1}^{n} \operatorname{Re}\left[w\left(t_{j}\right)\right] \mu_{1}\left(t_{j}\right)-\sum_{j=1}^{n} \operatorname{Im}\left[w\left(t_{j}\right)\right] \mu_{2}\left(t_{j}\right) \\
+c_{1} \sum_{j=1}^{n} \operatorname{Im}\left[w\left(t_{j}\right)\right]+c_{2} \sum_{j=1}^{n} \operatorname{Re}\left[w\left(t_{j}\right)\right] \\
=-\sum_{j=1}^{n} \operatorname{Re}\left[w\left(t_{j}\right)\right] \gamma_{2}\left(t_{j}\right)-\sum_{j=1}^{n} \operatorname{Im}\left[w\left(t_{j}\right)\right] \gamma_{1}\left(t_{j}\right)
\end{aligned}
$$

and

$$
\begin{array}{r}
-\sum_{j=1}^{n} \operatorname{Im}\left[w\left(t_{j}\right)\right] \mu_{1}\left(t_{j}\right)-\sum_{j=1}^{n} \operatorname{Re}\left[w\left(t_{j}\right)\right] \mu_{2}\left(t_{j}\right) \\
+c_{1} \sum_{j=1}^{n} \operatorname{Re}\left[w\left(t_{j}\right)\right]-c_{2} \sum_{j=1}^{n} \operatorname{Im}\left[w\left(t_{j}\right)\right] \\
=\sum_{j=1}^{n} \operatorname{Im}\left[w\left(t_{j}\right)\right] \gamma_{2}\left(t_{j}\right)-\sum_{j=1}^{n} \operatorname{Re}\left[w\left(t_{j}\right)\right] \gamma_{1}\left(t_{j}\right) .
\end{array}
$$

Discretizing the conditions (56) and (58), we obtain

$$
\begin{gathered}
\frac{1}{n} \sum_{j=1}^{n} \phi\left(t_{j}\right) \mu_{1}\left(t_{j}\right)-c_{2}=\frac{-1}{n} \sum_{j=1}^{n} \phi\left(t_{j}\right) \gamma_{2}\left(t_{j}\right), \\
\frac{-1}{n} \sum_{j=1}^{n} \phi\left(t_{j}\right) \mu_{2}\left(t_{j}\right)-c_{1}=\frac{-1}{n} \sum_{j=1}^{n} \phi\left(t_{j}\right) \gamma_{1}\left(t_{j}\right) .
\end{gathered}
$$

Hence, the Robin problem in an unbounded simply connected region we obtain a $2 n \times(2 n+2)$ linear 
system, including the two conditions (39) gives a $(2 n+2) \times(2 n+2)$ linear system. Adding the two equations obtained from (64) and (65) makes a $(2 n+4) \times(2 n+2)$ linear system. Finally, the two more equations from (66) and (67) are added to give a $(2 n+6) \times(2 n+2)$ linear system.

The solution from the system can be used to calculate the boundary values of the analytic function $f_{n}(\eta(t))$ by using the following formula

$$
f_{n}(\eta(t))=(-\varphi+\mathrm{i} \psi) \mathrm{e}^{-\mathrm{i} \kappa(t)} .
$$

The approximate interior values of the function $f(z)$ are calculated by the Cauchy integral formula

$$
f(z)= \begin{cases}\frac{\frac{1}{2 \pi \mathrm{i}} \int_{0}^{2 \pi} \frac{f(\eta)}{\eta-z} \mathrm{~d} \eta}{\frac{1}{2 \pi \mathrm{i}} \int_{0}^{2 \pi} \frac{1}{\eta-z} \mathrm{~d} \eta}, & z \in \Omega^{+}, \\ \frac{f(\infty)+\frac{1}{2 \pi \mathrm{i}} \int_{0}^{2 \pi} \frac{f(\eta)}{\eta-z} \mathrm{~d} \eta}{1+\frac{1}{2 \pi \mathrm{i}} \int_{0}^{2 \pi} \frac{1}{\eta-z} \mathrm{~d} \eta}, & z \in \Omega^{-} .\end{cases}
$$

Here, $f(\infty)=0$ for unbounded region $\Omega^{-}$. Numerically, (69) has the advantage that the denominator compensates for the error in the numerator ${ }^{21}$. The integrals (69) are approximated by the trapezoidal rule. The respective discretization formula for interior and exterior regions are

$$
f_{n}(z)= \begin{cases}\frac{\sum_{i=1}^{n} \frac{f_{n}\left(\eta\left(t_{i}\right)\right) \dot{\eta}\left(t_{i}\right)}{\eta\left(t_{i}\right)-z}}{\sum_{i=1}^{n} \frac{\dot{\eta}\left(t_{i}\right)}{\eta\left(t_{i}\right)-z}}, & z \in \Omega^{+}, \\ \frac{\sum_{i=1}^{n} \frac{f_{n}\left(\eta\left(t_{i}\right)\right) \dot{\eta}\left(t_{i}\right)}{\eta\left(t_{i}\right)-z}}{i n+\sum_{i=1}^{n} \frac{\dot{\eta}\left(t_{i}\right)}{\eta\left(t_{i}\right)-z}}, & z \in \Omega^{-} .\end{cases}
$$

\section{NUMERICAL EXAMPLES}

We consider some examples in the interior and exterior simply connected regions for Robin problem with the boundary condition $l(t)$ as (24).

Example 1 We consider the Laplace equation $\Delta u(z)=0$, where $z \in \Omega^{+}$bounded by a circle $\Gamma$ defined by $\eta(t)=\mathrm{e}^{\mathrm{i} t}, 0 \leqslant t \leqslant 2 \pi$. In condition (1) we choose $\alpha(t)=1-0.2 \sin t, \beta(t)=1$, and

$$
\begin{array}{r}
l(t)=(1-0.2 \sin t)(\cosh (\sin t) \sin (\cos t)-2) \\
+\cos t \cos (\cos t) \cosh (\sin t) \\
+\sin t \sin (\cos t) \sinh (\sin t)
\end{array}
$$

with an exact solution

$$
u(\eta(t))=\operatorname{Re}[f(\eta(t))]=\cosh (\sin t) \sin (\cos t)-2,
$$

Table 1 The errors $\left\|u(\eta(t))-u_{n}(\eta(t))\right\|_{\infty}$ on boundary $\Gamma$ for Example 1.

\begin{tabular}{rc}
\hline$n$ & $\left\|u(\eta(t))-u_{n}(\eta(t))\right\|_{\infty}$ \\
\hline 32 & $2.20 \times 10^{-3}$ \\
64 & $1.31 \times 10^{-4}$ \\
128 & $7.92 \times 10^{-6}$ \\
256 & $4.79 \times 10^{-7}$ \\
512 & $2.96 \times 10^{-8}$ \\
1024 & $1.84 \times 10^{-9}$ \\
\hline
\end{tabular}

Table 2 Absolute error $\left|f(z)-f_{n}(z)\right|$ at selected points on $\Omega$ for Example 1 .

\begin{tabular}{rcccc}
\hline$n$ & $0+0 \mathrm{i}$ & $0.3-0.5 \mathrm{i}$ & $-0.1+0.2 \mathrm{i}$ & $-0.3+0.6$ \\
\hline 32 & $3.2 \times 10^{-4}$ & $8.6 \times 10^{-4}$ & $3.2 \times 10^{-4}$ & $4.8 \times 10^{-4}$ \\
64 & $9.4 \times 10^{-6}$ & $4.0 \times 10^{-5}$ & $1.1 \times 10^{-5}$ & $2.7 \times 10^{-5}$ \\
128 & $2.7 \times 10^{-7}$ & $2.2 \times 10^{-6}$ & $4.5 \times 10^{-7}$ & $1.6 \times 10^{-6}$ \\
256 & $7.9 \times 10^{-9}$ & $1.3 \times 10^{-7}$ & $2.0 \times 10^{-8}$ & $9.5 \times 10^{-8}$ \\
512 & $3.6 \times 10^{-10}$ & $7.6 \times 10^{-9}$ & $1.1 \times 10^{-9}$ & $5.8 \times 10^{-9}$ \\
1024 & $2.7 \times 10^{-11}$ & $4.7 \times 10^{-10}$ & $6.0 \times 10^{-11}$ & $3.6 \times 10^{-10}$ \\
\hline
\end{tabular}

where $f(z)=\sin z-2$.

This yields the exact values $c_{1}=0$ and $c_{2}=$ 1.1585. For this example, $A(t)=\mathrm{e}^{\mathrm{i}(t+0.2 \cos t-0.2)}$, $t \in[0,2 \pi]$. The integrals (31), (32), (33), and (34) are calculated by the Gauss-Legendre rule with 256 nodes.

Table 1 lists the maximum error norms $\left\|u(\eta(t))-u_{n}(\eta(t))\right\|_{\infty}$, where $n$ is the number of nodes and $u_{n}(\eta(t))$ is the numerical approximation of $u(\eta(t))$ based on our method. The errors $\left\|f(z)-f_{n}(z)\right\|$ at some selected points are listed in Table 2. The absolute errors $\left|u(z)-u_{n}(z)\right|$ for selected points in the entire domain are plotted in Fig. 3. Fig. 4 shows the surface plot of $u_{n}(z)$ with $n=1024$.

Example 2 We consider the Laplace equation $\Delta u(z)=0$, where $z \in \Omega^{-}$, an unbounded domain with boundary $\Gamma$ defined by $\eta(t)=\mathrm{e}^{-\mathrm{i} t}, 0 \leqslant t \leqslant 2 \pi$. For condition (1), we choose $\alpha(t)=1+0.5 \cos t$ and $\beta(t)=1$. The function $l(t)=2 \cos t+0.5 \cos ^{2} t$ is obtained by choosing an exact solution

$$
u(\eta(t))=\operatorname{Re}[f(\eta(t))]=\cos t,
$$

where $f(z)=1 / z$. This yields the exact values, $c_{1}=$ 0 and $c_{2}=-1$. For this example, $A(t)=\mathrm{e}^{\mathrm{i}(t+0.5 \sin t)}$, $t \in[0,2 \pi]$. The integrals (31), (32), (33), and (34) are calculated by the Gauss-Legendre rule with 256 nodes.

Table 3 lists the maximum error norms $\left\|u(\eta(t))-u_{n}(\eta(t))\right\|_{\infty}$, where $n$ is the number of 


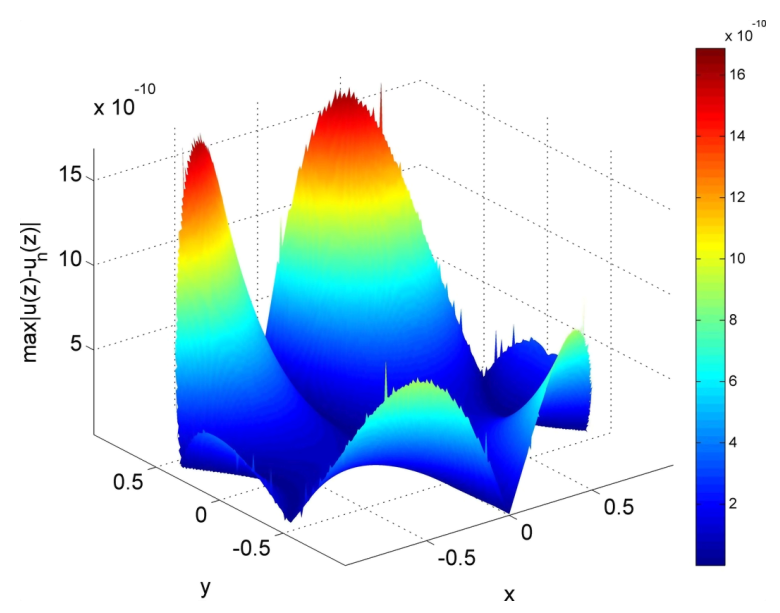

Fig. 3 The absolute error $\left|u(z)-u_{n}(z)\right|$ for the entire domain with $n=1024$ for Example 1.

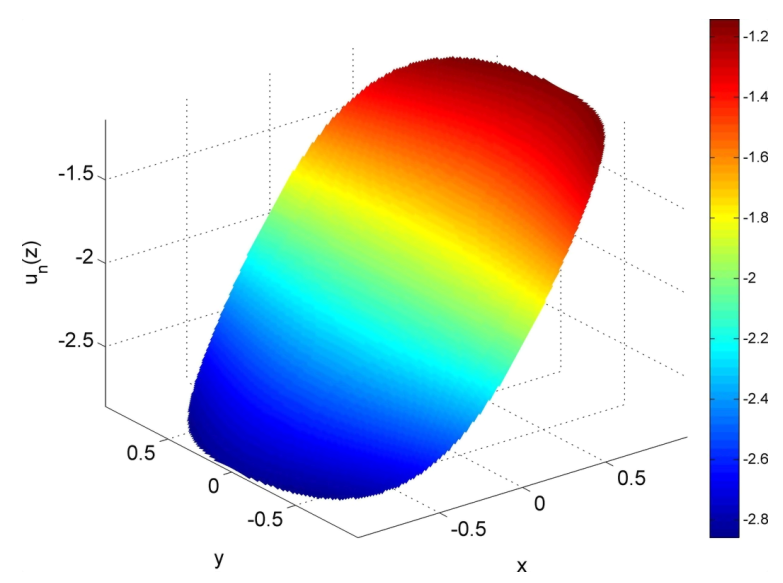

Fig. 4 The surface plot of $u_{n}(z)$ for Example $1(n=1024)$.

nodes and $u_{n}(\eta(t))$ is the numerical approximation of $u(\eta(t))$ based on our method. The absolute errors $\left|f(z)-f_{n}(z)\right|$ at some selected points are listed in Table 4. The absolute errors $\left|u(z)-u_{n}(z)\right|$ on the entire domain are plotted in Fig. 5. Fig. 6 shows the surface plot of $u_{n}(z)$ with $n=512$.

Table 3 The errors $\left\|u(\eta(t))-u_{n}(\eta(t))\right\|_{\infty}$ on boundary $\Gamma$ for Example 2.

\begin{tabular}{rc}
\hline$n$ & $\left\|u(\eta(t))-u_{n}(\eta(t))\right\|_{\infty}$ \\
\hline 32 & $9.54 \times 10^{-4}$ \\
64 & $4.29 \times 10^{-5}$ \\
128 & $1.84 \times 10^{-6}$ \\
256 & $1.32 \times 10^{-7}$ \\
512 & $1.00 \times 10^{-8}$ \\
\hline
\end{tabular}

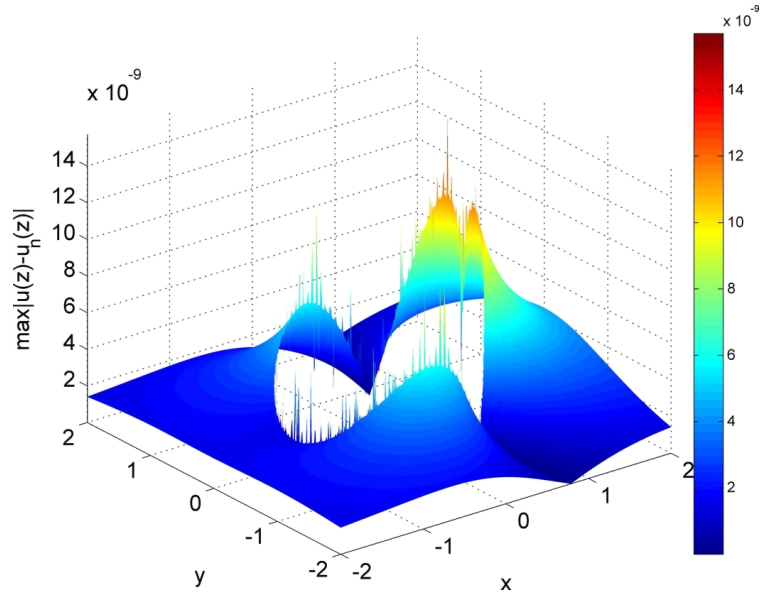

Fig. 5 The absolute error $\left|u(z)-u_{n}(z)\right|$ for the entire domain with $n=512$ for Example 2 .

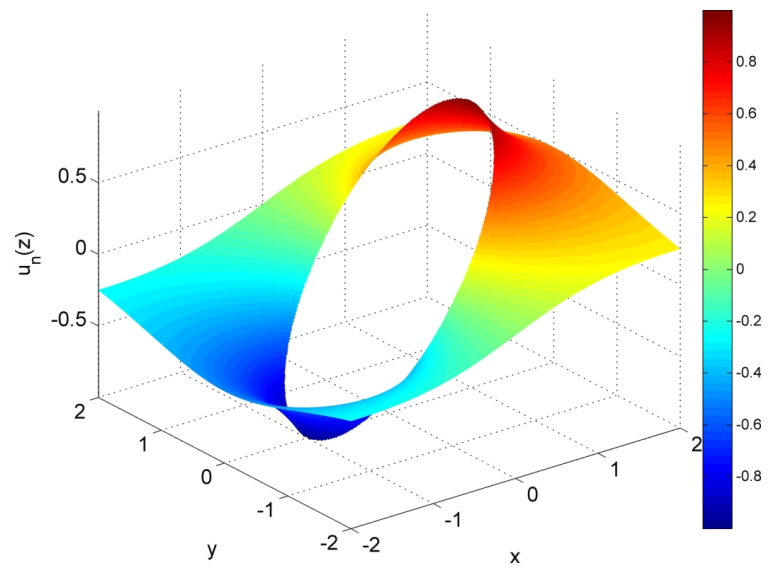

Fig. 6 The surface plot of $u_{n}(z)$ for Example $2(n=512)$.

\section{CONCLUSIONS}

In this paper, we have developed a method for solving some interior and exterior Robin problems in simply connected regions with smooth boundaries using a combination of integral equations, differential equations, normalizing conditions, and unknown constants $c_{1}, c_{2}$. The method works for

Table 4 Absolute errors $\left|f(z)-f_{n}(z)\right|$ at selected points on $\Omega$ for Example 2 .

\begin{tabular}{rccc}
\hline$n$ & $z=-1.3+1.1 \mathrm{i}$ & $z=-1.2+1.2 \mathrm{i}$ & $z=-1-1.5 \mathrm{i}$ \\
\hline 32 & $5.49 \times 10^{-5}$ & $7.36 \times 10^{-5}$ & $7.85 \times 10^{-5}$ \\
64 & $6.15 \times 10^{-6}$ & $7.29 \times 10^{-6}$ & $8.34 \times 10^{-6}$ \\
128 & $4.75 \times 10^{-7}$ & $5.53 \times 10^{-7}$ & $6.16 \times 10^{-7}$ \\
256 & $3.16 \times 10^{-8}$ & $3.66 \times 10^{-8}$ & $4.06 \times 10^{-8}$ \\
512 & $2.02 \times 10^{-9}$ & $2.33 \times 10^{-9}$ & $2.59 \times 10^{-9}$ \\
\hline
\end{tabular}


a special case of Robin problem for which $\alpha(t)$ and $\beta(t)$ satisfy the condition $\kappa(2 \pi)-\kappa(0)=2 \pi$. The integral equations are discretized by the Nyström method with trapezoidal rule and Wittich's method, while the differential equation is discretized by the five-point central difference method. The presented numerical results illustrate that the proposed method can be used to produce approximations of high accuracy. More work is required to extend the presented method to multiply connected regions. For an extension of the method in Ref. 12 to multiply connected regions, see Ref. 13.

Acknowledgements: The authors would like to thank the Malaysian Ministry of Education and Research Management Centre, Universiti Teknologi Malaysia for the partial funding through the research vote (R.J130000. 7809.4F637). We are also indebted to Mohamed MS Nasser for helpful discussions. The authors would like to thank the referees for their helpful comments and suggestions which improved the presentation of the paper.

\section{REFERENCES}

1. Heiskanen WA, Moritz H (1967) Physical Geodesy, Freeman, San Francisco.

2. Fang W, Zeng S (2009) Numerical recovery of Robin boundary from boundary measurements for the Laplace equation. J Comput Appl Math 224, 573-80.

3. Gustafson K, Abe T (1998) The third boundary condition-was it Robin's? Math Intell 20, 63-71.

4. Sneddon IN (1966) Mixed Boundary Value Problems in Potential Theory, North-Holland, Amsterdam.

5. Gakhov FD (1966) Boundary Value Problem, Pergamon Press, Oxford.

6. Duffy DG (2008) Mixed Boundary Value Problems, CRC Press, New York.

7. Seslija M, Perunicic B (2009) Extrapolation-based approach to optimization with constraints determined by the Robin boundary problem for the Laplace equation. In: XXII International Symposium on Information, Communication and Automation Technologies, IEEE, Bosnia, pp 1-7.

8. Alipour M, Vali MA (2015) Appling homotopy analysis method to solve optimal control problems governed by Volterra integral equations. J Comput Sci Comput Math 5, 41-7.

9. Wegmann R, Murid AHM , Nasser MMS (2005) The Riemann-Hilbert problem and the generalized Neumann kernel. J Comput Appl Math 182, 388-415.

10. Wegmann R, Nasser MMS (2008) The RiemannHilbert problem and the generalized Neumann kernel on multiply connected regions. J Comput Appl Math 214, 36-57.

11. Hamzah ASA, Nasser MMS (2012) A numerical method for solving the Robin problem. In: Murid
AHM, Yaacob Y (eds) Recent Advances in Applied and Computational Complex Analysis, Penerbit UTM Press.

12. Hamzah ASA, Murid AHM, Nasser MMS (2013) Boundary integral equations with the generalized Neumann kernel for Robin problem in simply connected region. Int J Appl Math Stat 44, 8-20.

13. Al-Shatri SHH, Murid AHM, Ismail M, Muminov MI (2016) Solving Robin problems in multiply connected regions via an integral equation with the generalized Neumann kernel. Bound Value Probl 2016, 91.

14. Petrila T (1999) Complex value boundary elements methods (CVBEM) for some mixed BVP. Stud Univ Babeş-Bolyai Inform 44(2), 37-42.

15. Mattheij RMM, Rienstra SW, ten Thije Boonkkamp JHM (2005) Partial Differential Equations: Modeling, Analysis, Computation, SIAM, Philadelphia.

16. Nasser MMS (2009) The Riemann-Hilbert problem and the generalized Neumann kernel on unbounded multiply connected regions. Univ Res J Ibb Univ 20, 47-60.

17. Nasser MMS, Murid AHM, Ismail M, Alejaily EMA (2011) Boundary integral equations with the generalized Neumann kernel for Laplace's equation in multiply connected regions. Appl Math Comput 217, 4710-27.

18. Atkinson KE (1997) The Numerical Solution of Integral Equations of the Second Kind, Cambridge Univ Press, Cambridge.

19. Gaier D (1965) Konstruktive Methoden der Konformen Abbildung, Springer, Berlin.

20. Abramowitz M, Stegun IE (1965) Handbook of Mathematical Functions, with Formulas, Graphs, and Mathematical Tables, Dover, New York.

21. Helsing J, Ojala R (2008) On the evaluation of layer potentials close to their sources. J Comput Phys 227, 2899-921. 